Hautecouverture, J.-C., Grégori, N., Brassac, Ch. (2007). Appropriation d'une plate-forme de Coopération par des enfants en cadre scolaire. Revue Européenne de Psychologie Appliquée, Vol. 57, $\mathrm{N}^{\circ} 1,1-16$. [psycINFO n 1162-9088] [www.springerlink.com ; DOI: 10.1007/s00146-005-0019-0]

\title{
APPROPRIATION D'UNE PLATE-FORME DE COOPÉRATION PAR DES ENFANTS EN CADRE SCOLAIRE
}

\author{
Jean-Charles Hautecouverture - Nicolas Grégori - Christian Brassac \\ Équipe Codisant-LabPsyLor - Université Nancy 2 \\ BP 33-97, 54015 Nancy Cedex \\ Prenom.Nom@univ-nancy2.fr
}

\begin{abstract}
Résumé
Les enseignants en école primaire utilisent de plus en plus fréquemment des dispositifs informatiques dans le cadre de leurs activités pédagogiques. Si ces dispositifs sont souvent utilisés individuellement par les élèves, ils donnent aussi lieu à des pratiques collaboratives. C'est le cas des plates-formes de coopération qui mettent en contact les élèves via des réseaux informatiques. Ces plates-formes sont développées par les chercheurs en informatique, dans le domaine du TCAO (Travail Collaboratif Assisté par Ordinateur). Le développement de tels collecticiels n'est pas qu'un problème technique; il se heurte à la question de l'appropriation par les acteurs concernés (élèves et enseignants) des fonctionnalités techniques offertes par ces outils de communication. Aborder de front les questions liées à cette constatation classique nécessite de travailler dans un cadre interdisciplinaire. En effet, l'étude de l'activité des élèves, sujets humains en situation d'interactions médiées par des instruments numériques, relève d'une approche que des chercheurs en psychologie (ici psychologie sociale des processus cognitifs collaboratifs) peuvent mener à bien en mettant en œuvre des méthodes et des catégories permettant une analyse des difficultés que les acteurs rencontrent. Nous montrerons comment ces analyses forment la trame de l'articulation des deux domaines de recherche concernés et conduisent pratiquement à la conception et l'élaboration des prototypes successifs de la plate-forme.
\end{abstract}

Mots-clés. Appropriation des TIC, Analyse des interactions, Ergonomie, Culture, Scénario pédagogique.

\begin{abstract}
The idea of using computers in primary school to enhance or even transform learning is more and more frequent. Even if these devices are often used in an individual way, they also offer a collaborative praxis. This is possible by cooperation platforms that link the learners through informatical network. Those platforms are developed by researchers of computing in the field of CSCW (Computer-Supported Collaborative Work). It is not only a technical problem to develop those software. On the user's side (children and teachers) there is some kind of toughness for the appropriation of the technical possibilities of these tools of communication. In order to tackle these questions, it is required to work in an interdisciplinary framework. Indeed, it is possible to study the activity of the learners in interaction, mediated by digital tools, with an approach of the research in psychology (here social psychology of cognitive processes in collaborative settings). Implementing methods and categories permit the analyse of the problems of the actors to master the platform functioning. In this paper, we will describe how these analyses form the framework of the articulation between the two fields of research and how they lead to the design and the development of successive prototypes of the platform.
\end{abstract}

Keywords. Appropriation of Information and Communication Technology (ICT), Analyse of Interaction, Ergonomics, Culture, Pedagogical Scenario. 


\section{Introduction}

Les dispositifs informatiques sont de plus en plus présents dans les établissements scolaires. Ce sont des outils pédagogiques parmi d'autres, que les enseignants et les élèves utilisent dans des cadres individuels aussi bien que collectifs, comme c'est le cas dans l'étude que nous présentons ici. Nous nous intéresserons en effet à des situations de création de documents où les élèves font équipe avec des camarades de la même classe et avec des enfants d'autres classes (qu'ils ne rencontreront physiquement qu'à la fin du projet). Le dispositif qui est en jeu est un collecticiel à visée pédagogique, une plate-forme de coopération. Cet équipement permet aux élèves de créer, diffuser et partager des fichiers, autant de fonctionnalités qui sont classiquement développées par les chercheurs du domaine du travail coopératif assisté par ordinateur (TCAO). Si leur implémentation est relativement bien maîtrisée par les concepteurs de collecticiels, leur appropriation par les élèves n'est en revanche pas sans poser des difficultés. Cette appropriation malaisée est doublement caractérisée. Si les enfants rencontrent des difficultés cognitives de manipulation de l'outil informatique en tant que tel, ils peinent aussi à en saisir leurs fonctions sociales.

Dans un premier temps, les élèves sont confrontés à des questions de type : Comment enregistrer un texte qu'ils viennent de rédiger? Comment utiliser les procédures de publication de ce fichier sur un espace commun avec d'autres élèves ? Comment récupérer un document déposé par d'autres dans tel ou tel espace ? Ils sont là dans un domaine d'usage des ressources techniques de la plate-forme. En fait, on constate assez rapidement que les enfants acquièrent relativement aisément ces fonctionnalités de la plate-forme. Ils apprennent en peu de temps à connaître et à manipuler les ressources offertes par le dispositif (l'existence des espaces disponibles, les actions qui permettent de passer des uns aux autres, etc.). Dans un second temps, néanmoins, ces enfants, qui sont rappelons-le en situation coopérative, éprouvent des difficultés à mobiliser ces fonctions dans le rapport qu'ils entretiennent aux autres usagers de cette plate-forme. Tel élève, par exemple, bien que sachant publier un fichier (c'est-à-dire le faire passer de son espace privé à l'espace commun), n'arrive pas à anticiper le fait que ses correspondants (ses 'équipiers' en l'occurrence) peuvent avoir besoin de savoir qu'il va le publier ; il peine à intégrer sa connaissance 'technique' du dispositif dans sa façon d'interagir avec les autres acteurs. Autrement dit, la constatation évoquée ici est qu'au-delà de son usage technique initialement problématique, c'est la mobilisation de la plate-forme à fins de collaboration sociale qui soulève la plus grande difficulté. Telle est la constatation que nous faisons, nous chercheurs en psychologie, dans le cadre 'applicatif' de l'appropriation d'un dispositif numérique par des sujets humains dans un cadre de travail coopératif.

Cette étude s'inscrit dans un projet interdisciplinaire, nommé Coopéra ${ }^{1}$, qui trouve son origine dans les considérations suivantes. Les dispositifs informatiques de coopération ne sont pas uniquement des objets techniques permettant la numérisation des connaissances. Ce sont aussi, et surtout, des objets sociaux qui ont une histoire (de leur conception à leur usage), qui ont une fonction sociale (de médiation inter-agents) et qui ont une 'valeur cognitive'. Toutes choses dont les ingénieurs technologues, modélisateurs et développeurs de cette machinerie informationnelle, sont maintenant bien conscients, eux qui en appellent de façon récurrente à ce 'fameux' facteur humain. Il est clair que les chercheurs qui font profession de développer ces plates-formes de coopération ne peuvent faire l'économie d'une réflexion approfondie relative à l'activité cognitive de l'humain. Pourquoi ? Parce que les plates-formes sont destinées à être mobilisées par des acteurs! L'utilisation efficace d'une telle plate-forme ne peut pas ne se mesurer qu'en termes d'optimisation des procédures de gestion de fichiers par

\footnotetext{
${ }^{1}$ Coopéra est un projet RIAM (Réseau d'Innovation en Audio-visuel et Multimédia) financé par le CNC (Centre National du Cinéma) dépendant du Ministère de la Culture (2002/2004). http://www.riam.org/
} 
exemple. L'appropriation effective par les acteurs concernés de ce type de procédure doit faire partie du mode de validation de la plate-forme. Il en résulte que le psychologue qui étudie l'usage du dispositif, par les acteurs, en situation naturelle, doit intervenir tôt dans le processus de conception. Il y a là, dans ce cadre de conception assisté par l'usage, place pour un travail collaboratif entre informaticiens et spécialistes des activités cognitives humaines.

Notre point de départ de psychologues au sein de ce projet s'appuie donc sur le fait que l'outil numérique est un dispositif socio-technique. Il s'agit pour nous d'approcher son appropriation par les intéressés (élèves et enseignants); nous travaillerons à la fois sur la façon dont ce dispositif est mobilisé, manipulé, utilisé par les enfants, sur son utilité dans la mise en place par les enseignants de scénarios pédagogiques pertinents pour les élèves, et aussi sur son acceptabilité culturelle par les différents acteurs concernés.

Nous présenterons dans un premier temps notre méthodologie d'élaboration des données obtenues au sein de séances de travail. Dans un second temps, nous présenterons la problématique sur laquelle se fonde notre travail. Nous exposerons ensuite l'analyse empirique de deux moments-clé de deux séances de travail collaboratif mettant en scène deux enfants. Ces deux séances sont séparés par un travail d'animation motivé par un déficit repéré dans l'usage initial du dispositif. La discussion qui suivra porte sur le fait que cette animation non seulement répond à une nécessité pédagogique mais qu'elle apporte des remèdes adaptés au déficit mentionné ci-dessus. Nous conclurons en revenant sur la pertinence de la démarche interdisciplinaire pour proposer un mode de remédiation à cette difficulté d'appropriation du dispositif numérique par des élèves collaborant dans un cadre scolaire.

\section{Un point méthodologique sur la constitution des situations d'observations et d'analyse des usages}

\subsection{Une approche d'inspiration ethnographique}

Notre méthode de travail est proche de celle du CSCW (Computer Supported Cooperative Work), où il est courant d'adopter une approche ethnographique de l'activité (Baker, Greenberg,\& Gutwin, 2002 ; Cardon, 1997; Dourish, 2003). Il s'agit d'analyser les environnements de travail, constitués de personnes et d'artefacts. L'objectif est d'appréhender le rôle de l'environnement dans les productions et dans les modes de coopération, en considérant que les situations et les actions sont co-déterminées en permanence. Ainsi, les usages se construisent dans un environnement social incluant à la fois les comportements verbaux et non-verbaux, les relations sociales et la matérialité ambiante.

Plusieurs raisons nous guident dans ce choix. La première est épistémologique. Toute activité humaine est instrumentalisée par l'usage d'objets et s'appuie, de façon tout à fait liée, sur l'usage du langage. À travers ces objets et ces mots (Vygotsky, 1978), nous régulons et transformons l'environnement en même temps que nos propres conduites et, donc, nos relations aux autres. Il est donc important que les analyses produites rendent compte du poids de l'environnement (faits d'objets en particulier) et des relations sociales dans l'activité. La deuxième raison est pratique. Les situations que nous étudions dans Coopéra sont des situations dans lesquelles les interactions sociales sont fortes et dans lesquelles il est indéniable que le contexte influence l'activité. Nous ne cherchons pas à contrôler les multiples variables. Au contraire, c'est la situation dans toute sa complexité et dans sa singularité que nous voulons saisir. En outre, notre objectif n'est pas de comparer des groupes d'élèves dans des conditions expérimentales contrastées. C'est pourquoi on peut affirmer qu'une approche ethnométhodologique est plus appropriée dans notre cas qu'une approche de type psychologie expérimentale (Kaptelinin \& Nardi, 2003 ; Nardi, Gilbert, Mantei, \& McCarthy, 1993). 
En fait, nos références relèvent toutes d'une approche praxéologique de la conduite humaine, abordée principalement sous l'angle de ses déterminants cognitifs et actionnels. De notre point de vue de psychologues sociaux, ces références touchent à différentes postures d'origines sociologique, l'action située (Suchman, 1987; Conein \& Jacopin, 1994) et la théorie des acteurs réseaux (Latour, 1999; Callon, Lascousmes, \& Barthe, 2001), et psychologique, la théorie de l'activité (les néo-vygotskiens autour des travaux d'Engeström, Miettinen, \& Punamäki, 1999) et la cognition distribuée (Hutchins, 1995). Ces différentes postures se différencient sur bien des points (la place de l'objet, le concept d'acteur par exemple) mais elles se retrouvent toutes autour d'un point commun : aborder les processus cognitifs et actionnels de façon externaliste (versus mentaliste) et interactionniste. La démarche ethnographique que nous adoptons est également un point commun à toutes ces approches.

\subsection{Constitution de situations naturelles}

L'activité humaine transforme l'environnement en même temps qu'elle est transformée par ce même environnement, dans une sorte de couplage action-environnement. Pour l'étudier, il est illusoire de vouloir la caractériser à l'aide de paramètres expérimentaux. Il faut au contraire mettre en place des situations dans lesquelles des pratiques naturelles seront accomplies. Vygotsky (1978) propose une méthode expérimentale-génétique qui répond à cet objectif. Nous nous en inspirons en constituant des paires d'élèves qui travaillent dans leurs propres salles de classe sur des projets conçus par leurs instituteurs. Cela présente deux intérêts pour nous. D'une part, cela renvoie à la réalité de l'utilisation des technologies informatiques en France, d'autre part, cela génère des discussions entre élèves que nous pouvons analyser. Bien sûr, nous adaptons cette méthode aux technologies audio-visuelles actuelles qui nous permettent l'enregistrement de traces langagières et matérielles. Plus précisément, nous utilisons deux caméras, équipées de microphones. L'une est orientée vers les élèves. Elle enregistre les acteurs en train de se parler, de se mouvoir, etc. L'autre réalise une capture directe de l'écran d'ordinateur, en continu. Ainsi, il s'agit de traquer, là même où elles adviennent, les créations des sujets, autant de médiations que Vygotsky considère comme étayant l'engendrement des cognitions. Il s'agit de mener à bien une clinique de l'activité comme l'entend Clot (1999).

Nous observons également l'activité des enfants lors de leur utilisation de la plate-forme. Et même, nous intervenons de telle sorte qu'ils puissent verbaliser leurs actions, leurs intentions d'action, leurs blocages, leurs désaccords, etc. Verbalisations que nous complétons par des temps d'auto-confrontations, à la façon de Clot, Faïta, Fernandez, \& Scheller (2001). Il s'agit alors de compléter les observations pratiquées (enregistrements vidéo et notes) par une séquence de travail au cours de laquelle les enfants sont confrontés à leur propre activité filmée, qu'ils sont invités à commenter.

Enfin, nous nous inscrivons dans le cadre d'une conception participative, ce que nous avons déjà décrit dans Godart et al. (2003) et dans Hautecouverture et al. (2003, 2004). Notre souci est de faire en sorte que les usagers finaux (élèves et instituteurs) soient des acteurs de la conception et pas simplement des testeurs de logiciels. Autrement dit, nous travaillons à la fois sur la place symbolique des usagers (quel statut leur donner dans le cycle de conception) et sur leur place physique (quand les faire intervenir dans ce cycle), voir Grégori (2002). En effet, c'est dans la confrontation entre usagers etéquipe de conception que l'usage est exprimé puis traduit en caractéristiques techniques. Les conséquences sur le protocole d'observation sont les suivantes. Les usagers sont immédiatement confronté au logiciel, malgré les insuffisances ergonomiques soupçonnées. Dans le cas du projet Coopéra, notons que ces insuffisances ne sont pas nécessairement des caractéristiques du logiciel mais qu'elles sont liées à son utilisation dans le contexte particulier d'une salle de classe. En outre, dire des 
usagers qu'ils sont considérés comme des acteurs/partenaires dans la conception, c'est affirmer que les développements techniques sont une construction conjointe entre équipes de développement et usagers. Nous n'attendons pas des usagers qu'ils livrent des solutions, ce qu'on peut parfois reprocher à la sociologie de l'usage, comme nous ne demandons pas aux développeurs d'anticiper des besoins, par ailleurs difficiles à définir sans confrontation réelle à l'outil. C'est bien sûr ici que les verbalisations des enfants ainsi que l'analyse de l'usage des différents prototypes de la plate-forme prennent leur sens pour le processus de conception.

\subsection{Analyse des corpus}

Les interactions sociales que nous enregistrons sont constituées de productions langagières et gestuelles et de manipulations d'objets (souris, écran, clavier, etc.). Ce sont donc les accomplissements des acteurs, au sens ethnométhodologique du terme, dont nous rendons compte. Pour analyser la partie langagière, nous recourons à la théorie des actes de langage (Searle \& Vanderveken, 1985; Vanderveken, 1988), même si, théoriquement, les principes de l'analyse conversationnelle de l'ethnométhodologie ne vont pas de pair avec ceux de l'analyse du discours, reposant sur les actes de langage (Levinson, 1983). Nous le faisons cependant car nous utilisons cette catégorie d'analyse dans une version dialogisée qui permet l'analyse de l'activité en cours (Brassac, 2004, Brassac \& Grégori, 2001a-b, 2003 ; Grégori, 2002). L'acte de langage est en effet un outil d'analyse intéressant car il renvoie à la fois à la dimension cognitive et à la dimension sociale des conversations. En ce qui concerne les productions gestuelles et les manipulations d'objet, nous analysons leur fonction dans la dynamique interactionnelle. Nous verrons que certains gestes, par exemple, sont associés à des actions langagières antérieures alors qu'il arrive que des gestes (un pointage, un 'clic' par exemple) précèdent l'expression orale afférente, voire constituent en eux-mêmes des formes sémiotiques qui génèrent la construction des significations. Dans le même ordre d'idée, la manipulation d'objet participe à la construction conjointes de ces significations. Autrement dit, nous analysons l'interaction entre les enfants comme un modelage de formes langagières, gestuelles et artefactuelles.

Cette position nous conduit à mettre en évidence le processus d'appropriation de la plateforme par les élèves, ainsi que le rôle du collecticiel dans ce processus : est-il un support favorable à sa réalisation? Est-il le seul facteur intervenant dans ce processus ?

\section{La coopération à distance : une question d'environnement informatique ?}

Les difficultés inhérentes à toutes situations de coopération à distance sont celles de la gestion même de l'activité coopérative. En effet, lorsque plusieurs utilisateurs distants travaillent sur le même document électronique, la mise en commun des productions de chacun se complexifie considérablement et le risque d'apparition de conflits informatiques entre les différentes versions de ce même document augmente fortement.

\subsection{Une plate-forme pour réguler l'activité coopérative}

Pour pallier les difficultés énoncées ci-dessus, l'équipe d'informaticiens partenaire dans le projet a développé une plate-forme informatique dont la spécificité est de permettre à plusieurs utilisateurs, en l'occurrence ici les élèves de CM1/CM2 d'établissements différents, non seulement de travailler sur un fichier commun, mais surtout, de réguler leur activité de production. Plus précisément, le mode de coopération permis par la plate-forme est caractérisé par deux types de conduites conscientes que les utilisateurs doivent effectuer les uns vis-à-vis des autres pour la rendre efficace : (i) prévenir l'ensemble des membres du projet lorsqu'un 
fichier est en cours de modification ; (ii) partager avec eux le fichier nouvellement modifié. À charge aux autres d'agir selon la situation rencontrée : (i) ne pas travailler sur le document commun lorsque celui-ci est en cours de modification, au risque d'engendrer un conflit; (ii) récupérer la nouvelle version du document mise à disposition, pour y apporter ensuite leurs propres modifications. La mise en œuvre d'une telle dynamique d'échange repose sur les deux dimensions qui fondent la spécificité de l'outil : le modèle de partage de fichiers et la visualisation de l'activité coopérative.

\subsubsection{Le modèle de partage de fichiers}

Le projet Coopéra repose sur ToxicFarm, une plate-forme de coopération développée par l'équipe Ecoo du Loria sur le modèle de divergence/convergence de Prospero (Dourish, 1998) dont une caractéristique essentielle est qu'elle donne lieu à un travail asynchrone. Les membres ne travaillent donc jamais simultanément sur le même fichier.

La clé du système repose sur le modèle de partage de fichiers (Figure 1), fondé sur l'approche "Long Transaction Model" (Feiler \& Downey, 1990). Il s'organise autour de trois espaces : (i) un espace local, présent sur l'ordinateur de chacun des membres du projet (c'est dans cet espace que les utilisateurs apportent les modifications à leurs propres fichiers) ; (ii) un espace privé, situé sur internet, pour chaque utilisateur (c'est dans cet espace qu'un utilisateur prévient les autres qu'il a modifié un fichier) ; (iii) un espace commun à tous les membres, situé également sur internet (c'est dans cet espace que le partage des fichiers s'effectue). Le principe est que les espaces local et privé des utilisateurs doivent être maintenus identiques, au moyen d'une synchronisation. Comme l'espace privé de chacun est sur internet, chaque membre peut savoir qu'un fichier a été modifié. Le partage d'un fichier s'organise en deux opérations. Lorsqu'un utilisateur a terminé un travail de création ou de modification d'un fichier et qu'il a synchronisé ses espaces local et privé, il publie la version du fichier dans l'espace commun pour le rendre accessible au groupe. Les autres membres sont alors responsables de la mise à jour de leur espace privé, qu'ils synchroniseront avec leur espace local, afin de consulter le fichier nouvellement modifié (ou créé) et d'y faire éventuellement des ajouts.

\section{"insérer figure 1 ici"}

Ainsi un élève qui vient de modifier un fichier opère une synchronisation entre son espace local et son espace privé puis le publie sur l'espace commun; les autres ont ainsi accès au résultat de son travail, la dernière version qu'il a réalisée. Par ailleurs, un élève souhaitant travailler sur un fichier placé dans l'espace commun, doit le mettre à jour dans son espace privé et le synchroniser dans son espace local ; il peut ainsi le travailler avant de revenir au cas précédent. On retrouve ces trois fonctions de base (synchronisation, publication et mise à jour) dans la Figure 1.

\subsubsection{La visualisation de l'activité coopérative}

La visualisation de l'activité coopérative (Figure 2) apparait dans le poste de pilotage, qui est l'interface dédiée à cet effet dans la version de la plate-forme analysée ici. Cette visualisation représente explicitement les différents espaces constitutifs du modèle de partage de fichiers, ainsi que les opérations pour le transfert des fichiers d'espace à espace (la synchronisation, la publication et la mise à jour). 
Un système d'activation d'icônes, combiné avec un code couleur, représente les différents espaces. Ce système permet aux utilisateurs de savoir à tout moment qui a fait quoi et qui est en train de faire quoi, ce qui leur permet ensuite d'agir en conséquence. Ainsi, lorsque toutes les icônes sont blanches, cela signifie qu'aucun fichier n'est en cours de modification ou n'a été publié. Lorsque l'icône représentant l'espace privé des autres est jaune, c'est le signe qu'un (ou plusieurs) membre(s) du projet sont en train d'apporter des modifications à un (ou plusieurs) fichier(s). La liste des membres et des fichiers en cours de modification figure alors dans une fenêtre située sous le code couleur. Dans cette situation les utilisateurs ont intérêt à vérifier que le (ou les) fichier(s) sur lesquels ils souhaitent travailler ne figurent pas dans cette liste. Si c'est le cas, alors les utilisateurs doivent attendre la fin des modifications pour y travailler afin de ne pas engendrer de conflit. Lorsque l'icône représentant l'espace commun est jaune, c'est le signe qu'un (ou plusieurs) membre(s) du projet partage(nt) ses (leurs) fichiers nouvellement modifiés. A charge pour les autres utilisateurs, donc notamment à moi, de les 'rapatrier'. Enfin, lorsque mon espace privé est en jaune, c'est le signe que j'ai fait des modifications sur un fichier ou que j'en ai créé un nouveau ; les autres en sont ainsi prévenus.

\subsection{Culture, ergonomie et scénario pédagogique}

Les fonctionnalités avancées de la plate-forme donnent la possibilité aux utilisateurs d'avoir "conscience" du travail collectif en train de se faire au fur et à mesure de son déroulement. Celle-ci se traduit par leur capacité à mener une pensée réflexive qui s'exprime par les deux conduites conscientes à réaliser pour éviter tout conflit informatique : prévenir et partager. Cependant, bien que «l'instrument [contienne] sous une forme spécifique, l'ensemble des rapports que le sujet peut entretenir avec la réalité sur et dans laquelle il permet d'agir, avec lui-même et avec les autres » (Rabardel, 1999, p. 262), le développement chez les utilisateurs de la conscience de soi et d'autrui souhaitée n'en est pas pour autant assuré. Selon Vygotsky, « la conscience est l'expérience vécue d'expériences vécues » (Vygotski cité par Clot, 2003, p. 15). La conscience est « rapport entre activités, liaison entre activités » (Clot, 2003, p. 12). Dès lors, les élèves ne peuvent accomplir les conduites conscientes visées (prévenir et partager) que s'ils ont déjà réalisé ce genre d'activité. Or, après enquête auprès des enseignants et consultation du 'Brevet Informatique et Internet', il ne semble pas que ce soit le cas. En d'autres termes, la possibilité d'une rupture entre l' 'idéologie' de l'outil et sa 'réception' par les acteurs doit être envisagée. Dans le cas que nous traitons, il s'agit d'une rupture entre le mode de coopération permis par la plate-forme et les modes de coopération constituant l'environnement culturel habituel des élèves de CM1/CM2 et des enseignants, modes 'classiques' intériorisés par les uns et les autres au cours de leurs interactions quotidiennes. L'acte de coopérer n'est pas un acte anodin. Il fait référence à des relations sociales entre individus, à des modes opératoires particuliers. Il est donc inscrit dans un environnement culturel complexe. Le développement de la plate-forme de coopération ne peut donc s'affranchir de cette dimension culturelle.

Si la conscience est pour chaque élève un instrument de médiation avec l'objet de son activité, avec les autres élèves et avec lui-même, elle est elle-même toujours médiatisée par un instrument : la plate-forme de coopération. Celle-ci est le support sur lequel reposent toutes les dynamiques de construction et de mobilisation de significations. Aussi, l'ergonomie du collecticiel constitue une seconde dimension intervenant dans le processus d'appropriation du collecticiel. Une troisième dimension à prendre en compte est le scénario pédagogique. Celuici est fondamental car il permet de situer l'action et, donc, de construire une situation d'observation naturelle. Il est essentiel que les situations de travail répondent et aux impératifs liés aux études d'usage et aux impératifs pédagogiques. Pour ce faire, trois exigences au moins doivent être prises en compte lors de leur construction. Premièrement, elles doivent 
favoriser la coopération, deuxièmement, la tâche à réaliser doit motiver les enfants et, troisièmement, les instituteurs doivent être impliqués dans le projet.

Ainsi, nous défendons l'hypothèse selon laquelle la mise en œuvre de la dynamique d'échange visée ne repose pas sur les seules propriétés techniques et ergonomiques d'un environnement NTIC, mais sur la mise en place d'un dispositif instrumental, c'est-à-dire sur l'ensemble des moyens pour permettre aux élèves de coopérer à distance : la plate-forme bien sûr, également des interventions de type "animations", dont l'objectif consiste à pallier la rupture culturelle liée au mode de coopération permis par la plate-forme, et aussi le scénario pédagogique.

Les analyses produites doivent ainsi permettre de caractériser l'usage de la plate-forme selon ces trois dimensions essentielles : culture, ergonomie et scénario pédagogique.

\section{Analyse d'usage de la plate-forme et préconisations de développement}

\subsection{Mise en place de projets de coopération réels pour les études d'usage}

Trois projets de coopération ont été réalisés, utilisant trois versions successives de plateforme. Chaque projet a été conçu en étroite collaboration avec les enseignants. Celui dont sont extraites les séquences analysées dans cet article est le troisième. Il porte sur la réalisation d'un magazine en ligne. Quatre classes d'écoles différentes y ont participé : deux écoles nancéiennes (Jules Ferry et Saint Pierre), une de Laxou (Louis Pergaud) et une de Toul (La Sapinière).

Nous avons mis en place une organisation propice à la coopération entre les classes : dans chaque classe, les élèves se sont regroupés par binôme (ou trinôme) et ont choisi un thème à traiter parmi douze thèmes proposés. Les binômes (ou les trinômes) des quatre écoles ayant choisi le même thème forment ainsi une équipe interclasse. En utilisant un forum disponible sur la plate-forme, les binômes (ou trinômes) de chaque équipe se sont ensuite réparti les rôles pour traiter leur sujet. Chaque équipe se trouvait effectivement en situation de coopération par le fait que les binômes (ou trinômes) de chaque équipe partageaient le même fichier d'une part, et qu'il y avait interdépendance entre leurs rôles d'autre part. Par exemple, une équipe a travaillé sur le peuple tibétain ; un binôme avait en charge un texte de présentation du Tibet et un autre devait en donner une illustration en utilisant soit une carte, soit un drapeau, etc.

La réalisation du magazine en ligne s'est déroulée de la manière suivante. Une première animation a été réalisée dans chaque classe avant les séances d'usage de la plate-forme en salle informatique. Cette animation avait pour fonction de présenter aux élèves, ainsi qu'aux enseignants, les principes de coopération permis par la plate-forme. Cette animation s'est déroulée avec tous les élèves en même temps. Nous avons préféré à des explications, l'établissement d'une mise en scène où les élèves en sont les acteurs et où les concepts sont matérialisés par des objets (dont certains de notre fabrication) que les enfants ont manipulés, déplacés au cours de l'animation. Puis, les élèves ont participé à trois séances informatiques successives, à raison d'une par semaine, au cours desquelles ils ont réalisé une première phase dans l'élaboration du magazine en ligne. Chaque séance a duré environ une heure. A l'issue de ces séances, nous avons réalisé une seconde animation, dont l'objectif était de faire prendre conscience aux élèves du mode de coopération permis par la plate-forme, sur la base de leurs activités réalisées lors des séances informatiques antérieures. Enfin, deux nouvelles séances d'utilisation du collecticiel ont eu lieu pour la seconde phase de la réalisation du magazine en ligne. Nous avons filmé les deux animations dans chaque classe et en ce qui concerne les séances informatiques, nous avons suivi quatre binômes (un pour chaque école). Si l'on ajoute les séances en auto-confrontation (qui n'ont pas pu être réalisées systématiquement pour 
chaque binôme, en raison de contraintes dans le planning des enseignants), la durée totale d'enregistrement à analyser est de trente heures.

\subsection{Entre usage du traitement de texte et du poste de pilotage}

Au long de leur activité coopérative, les élèves suivent la démarche présentée de façon détaillée ci-dessous (Tableau 1); elle se réalise en deux temps et est relative à deux types d'écran. Lorsqu'ils travaillent sur les fichiers, qu'ils les modifient ou qu'ils les créent, ils utilisent les applications logicielles dont ils disposent habituellement sur leurs machines (du type traitement de texte, tableur, logiciel de dessin, etc. ; noté TdT dans le tableau). Ce n'est que lorsqu'ils gèrent effectivement leur activité coopérative que les élèves utilisent la plateforme (noté PdP dans le tableau).

\section{" insérer tableau 1 ici "}

Ainsi, lorsque les enfants travaillent sur leurs fichiers, en vue de les partager, ils n'ont pas la plate-forme sous les yeux, puisqu'elle ne sert qu'au transfert des fichiers dans les espaces (espaces local, privé ou commun). En d'autres termes, l'état de l'écran ne leur fournit aucun moyen leur 'rappelant' les conduites qu'ils doivent adopter envers les autres : prévenir, dès le début de leur intervention, qu'ils vont apporter des modifications au fichier concerné et partager, à la fin de leur travail, le fait que ce fichier a été modifié. La mise en œuvre de ces conduites, fondamentales pour le bon usage de la plate-forme, repose dès lors sur les seuls modes de coopération intériorisés par les enfants. Nous allons voir que c'est ce type de question qui est en jeu dans la situation que nous allons analyser.

\subsection{Un binôme en cours d'usage}

Les deux séquences analysées portent sur le même type de situation. Un binôme, en l'occurrence deux élèves filles $\mathrm{C}$ et $\mathrm{M}$, a terminé d'apporter des modifications à un fichier travaillé par l'ensemble de l'équipe. Du point de vue de la dynamique coopérative, les deux élèves ont dû prévenir les autres lorsqu'elles étaient en plein travail. Elles doivent à présent partager leur fichier nouvellement modifié. Ainsi que nous allons le voir dans l'interaction reproduite ci-après [Extrait 1], ces deux élèves rencontrent quelques difficultés pour gérer cette activité coopérative. Difficultés qui proviennent directement du fait qu'elles n'ont pas intériorisé le mode de coopération supporté par la plate-forme. On remarquera en effet qu'elles ne font aucune référence à autrui au cours de leur activité, ni explicitement ni implicitement. Nous faisons alors l'hypothèse que ce déficit communicationnel n'est pas uniquement dû aux caractéristiques graphiques et fonctionnelles de la plate-forme, mais aussi, et peut-être surtout, au fait que le mode de coopération lui-même ne trouve pas d'écho dans les pratiques usuelles des enfants. En d'autres termes, l'environnement proposé ne s'inscrit pas dans un processus de banalisation (Mallein et Panisset, 1997). Nous dirons qu'il est culturellement en rupture. Pour remédier à ces difficultés, nous avons mis en place une animation, dont les fondements théoriques et la réalisation relèvent d'une perspective constructiviste. Après avoir présenté les principes de cette animation, nous en examinerons les apports à l'aide de l'analyse d'une deuxième séquence [Extrait 2]. 


\subsubsection{Séquence pré-animation : l'inscription des élèves dans une procédure d'utilisation sans référence à autrui}

Voici l'extrait conversationnel sur lequel va porter notre analyse. Les deux élèves $\mathrm{M}$ et $\mathrm{C}$ ont déjà utilisé le poste de pilotage $(\mathrm{PdP})$ lors de deux séances préalables. Au moment où commence cet extrait, elles viennent d'apporter des modifications à un fichier en utilisant un traitement de texte (TdT). Il s'agit maintenant de le partager avec les autres binômes de l'équipe c'est-à-dire d'utiliser le PdP pour le publier. L'animateur (An) est là pour observer la manière dont $\mathrm{M}$ et $\mathrm{C}$ vont agir pour atteindre cet objectif.

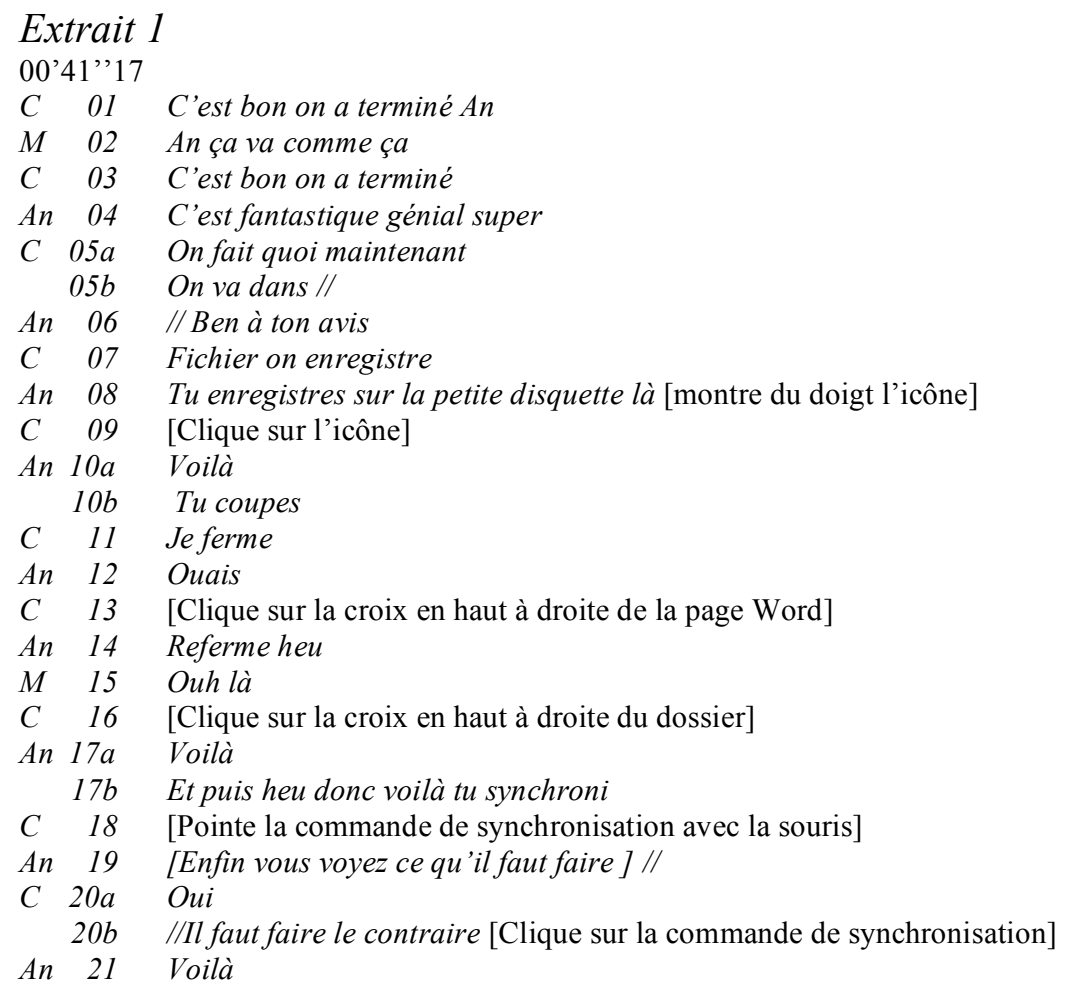

Un regard rapide sur cet extrait montre que, après avoir validé la fin de l'action précédente, les élèves $(\mathrm{C}$ et $\mathrm{M})$ ne savent pas vraiment ce qu'elles doivent réaliser à présent. L'animateur les aide alors à réaliser une suite de quatre actions : enregistrer le fichier, fermer le document, fermer le dossier qui contient le fichier modifié et, enfin, synchroniser. Les quatre échanges ${ }^{2}$ $\mathrm{E}[\mathrm{An06}-\mathrm{An} 10 \mathrm{a}], \mathrm{E}[\mathrm{An} 10 \mathrm{~b}-\mathrm{C} 13], \mathrm{E}[\mathrm{An} 14-\mathrm{An} 17 \mathrm{a}], \mathrm{E}[\mathrm{An} 17 \mathrm{~b}-\mathrm{C} 18]$ marquent ces quatre actions ; ils sont tous initiés par l'animateur et presque tous (les trois premiers) validés par lui (Figure 3). Cette suite d'actions structure une relation de type expert/élève, dans laquelle le premier indique au second la procédure à suivre et valide chaque action avant de passer à la suivante. La séquence s'achève sur une remarque de $\mathrm{C}$ selon laquelle, finalement, l'action à réaliser est « le contraire » d'une autre qu'ils ont réalisée plus tôt $(\mathrm{C} 20 \mathrm{~b})$. Nous y reviendrons, mais regardons ce qui se trame dans cet extrait.

\footnotetext{
${ }^{2}$ Nous utilisons là le vocabulaire du modèle hiérarchique et fonctionnel de la conversation (Roulet et al., 1985). Lorsque nous conversons, nous produisons des actes de langage qui forment des unités monologales appelées interventions (I), formant à leur tour des unités dialogales appelées échanges (E). A un niveau supérieur, ces échanges et/ou interventions forment de nouvelles interventions et échanges. Au niveau le plus subordonnant, ces structures donnent lieu à des transactions.
} 


\subsubsection{Absence de référence à autrui au cours du travail}

Les quatre premiers tours de parole concernent la fin d'une action de modification d'un fichier par $\mathrm{C}$ et $\mathrm{M}$. Rappelons que ce fichier est commun aux quatre binômes de l'équipe. L'important, ici, est de souligner que si $\mathrm{C}$ et $\mathrm{M}$ sont bien arrivées au terme de cette tâche de modification, telle qu'elle était définie au début de la séance, elles n'ont, en revanche, pas prévenu leurs partenaires distantes du fait qu'elles étaient en train de réaliser cette action. Or, en travaillant par devers elles, sans prévenir les autres, elles prennent le risque de créer une situation dite « potentiellement conflictuelle » au sein de la plate-forme. En effet, si plusieurs binômes modifient simultanément le même fichier, il y aura nécessairement un conflit de versions lors de la publication dans l'espace commun. Cette démarche, qui consiste à ne pas prendre ses partenaires en considération lors d'un travail sur un fichier commun, ne correspond donc pas au mode de coopération préconisé par la plate-forme. Nous avons noté dans le Tableau 2 ce que $\mathrm{M}$ et C (n')ont (pas) fait en regard de la démarche préconisée.

\section{" insérer tableau 2 ici »}

\subsubsection{Difficulté avec la procédure de travail}

Rappelons qu'après avoir modifié un fichier commun dans son espace local, il faut le porter dans l'espace commun, afin que les autres puissent le récupérer. Pour ce faire, il faut d'abord synchroniser son espace local et son espace privé, puis publier depuis l'espace privé vers l'espace commun. L'indécision de C (05a) peut renvoyer soit à une indécision d'ordre procédural (méconnaissance des opérations à réaliser pour partager leur fichier achevé), soit d'ordre épistémique (méconnaissance de la conduite à adopter vis-à-vis des autres membres de l'équipe). En fait, la situation est relativement informative en terme de procédure. Les élèves sont réunis à cette heure particulière et dans ce lieu particulier pour échanger des fichiers à l'aide de la plate-forme. Elles savent en effet que dans cette situation, elles devront utiliser la plate-forme pour 'faire' quelque chose. Elles le savent d'autant plus que c'est la troisième fois qu'elles sont dans cette situation. C l'exprime en ces termes : " on fait quoi maintenant » (05a) et poursuit immédiatement en ajoutant « on va dans » (05b). Comme C est interrompue dans sa proposition par An (06), nous faisons les deux hypothèses suivantes :

- ce lieu peut être tout à fait extérieur à la plate-forme (onglet menu, commande, etc.) ; auquel cas rien ne permet de dire que $\mathrm{C}$ fait référence à autrui ou non ;

- ce lieu est soit la fenêtre 'poste de pilotage' elle-même, soit un des espaces du poste de pilotage ; auquel cas, on peut faire l'hypothèse que $\mathrm{C}$ envisage de faire une action envers les autres membres de son équipe.

Cette alternative renvoie à une interrogation de fond sur le mode de travail des élèves. Soit elles sont capables de se projeter dans une dynamique coopérative (via la manipulation de la plate-forme ou plus précisément des espaces du poste de pilotage, c'est le cas b)), soit elles n'en sont pas capables. L'extrait 1 ne permet pas de trancher sur cette question mais on peut noter que, manifestement, $\mathrm{C}$ et $\mathrm{M}$ ne sont pas à l'aise avec la procédure de travail et que le fait d'être accompagné par un animateur les 'décharge' en quelque sorte de la responsabilité de la procédure. C'est ainsi que $\mathrm{C}$ et $\mathrm{M}$ se tournent vers $\mathrm{An}$ dès qu'elles ont achevé une action (E[C01-An04]). C'est ainsi, également, que leur premier geste est de se tourner vers l'animateur, celui qui sait, pour qu'il indique/valide la procédure à suivre. Ce réflexe pose notamment la question de la motivation des enfants. Nous y reviendrons en discussion.

De façon plus générale, la manipulation des outils informatiques (ici de bureautique) n'est pas encore naturelle chez $\mathrm{C}$ et $\mathrm{M}$. En effet, ce qu'il faut faire lorsqu'on a fini de travailler sur un 
fichier, c'est le partager. Mais ce n'est pas ça que C annonce. Elle ne dit pas que maintenant, il leur faut partager le fichier. Au contraire, elle reste dans une procédure liée à la manipulation du traitement de texte qu'elle utilise. En effet, alors que la question de An (06) pouvait être prise à divers niveaux, $\mathrm{C}$ propose d'enregistrer son fichier (07). Mais $\mathrm{C}$ n'est pas la seule à être mal à l'aise. L'expressif de $\mathrm{M}$ (15) est lui aussi très évocateur. Il agit ici comme une évaluation de l'action de $\mathrm{C}$ qui vient de fermer la fenêtre du traitement de texte, évaluation plutôt négative, puisque "ouh là là » évoque généralement un désagrément. En l'occurrence, c'est la lourdeur des opérations successives à réaliser que M exprime ainsi.

\subsubsection{L'animateur : entre gestion de la coopération et prescription de l'action}

Cet extrait marque donc une double hésitation de la part des enfants. Hésitation sur les actions à réaliser et hésitation sur la manipulation des outils informatiques. Intuitivement au moins, An ressent ce malaise. On l'a vu, sa première question (06) ne contraint pas l'action puisqu'elle est en miroir, renvoyant $\mathrm{C}$ à sa propre interrogation portant sur l'action à suivre. L'animateur est d'ailleurs tout à fait dans son rôle en agissant de la sorte. Il prend garde de ne pas engager $\mathrm{C}$ (et $\mathrm{M}$ ) dans une action à laquelle celle-ci n'aurait pas réfléchi, et qu'elle n'aurait donc ainsi pas comprise. Ainsi, il présuppose que $\mathrm{C}$ (et $\mathrm{M}$ ) peu(ven)t déterminer seule(s) ce qu'elle(s) doi(ven)t faire. Il présuppose aussi certainement que $\mathrm{C}$ va s'engager dans une procédure coopérative. Le fait est, cependant, que An va guider assez fortement $\mathrm{C}$ et $\mathrm{M}$ dans une suite d'opérations qui ne sont pas directement en lien avec la gestion de la dynamique coopérative. En quelque sorte, il se laisse enfermer par $\mathrm{C}$ et $\mathrm{M}$ dans une relation expert/novice, changeant alors de rôle. D'animateur, c'est-à-dire de gestionnaire d'une activité centrée sur la coopération, il devient prescripteur d'actions précises relatives à l'utilisation d'une machinerie informatique, comme le montre la suite d'échanges E[An06An10a], E[An10b-C13], E[An14-An17a], E[An17b-C18].

Ce glissement génère alors une confusion chez l'animateur, exprimée en 19 par une formulation pour le moins ambiguë («enfin vous voyez ce qu'il faut faire »), alors que ses interventions précédentes, celles qui guidaient les opérations de $\mathrm{C}$ et $\mathrm{M}$, étaient particulièrement précises. C'est ainsi qu'en 08 , il décrit l'action à réaliser au moyen d'une redondance parole/geste, dénommant l'icône d'enregistrement (la petite disquette) et la pointant du doigt. Par la suite, il continue de dire relativement clairement à $\mathrm{C}$ ce qu'elle doit faire : couper (10b), refermer (14), synchroni[ser] (17b). À l'opposé de ces formulations, l'énoncé 19 apparaît en retrait, comme si l'animateur regrettait d'être allé trop loin en $17 \mathrm{~b}$. C'est qu'il y a une différence de taille entre les prescriptions $08,10 \mathrm{~b}$ et 14 d'une part et $17 \mathrm{~b}$ d'autre part. Les premières sont relatives à l'utilisation du traitement de texte et du système d'exploitation, environnements qui ne font pas l'objet de la formation à la plate-forme Coopéra. L'indication $17 \mathrm{~b}$, en revanche, concerne directement l'animation puisqu'elle renvoie à la synchronisation, une des trois opérations au cœur du système de partage de fichiers. Subrepticement, donc, An s'est laissé envahir par le rôle d'expert qu'il a endossé, ce qui le conduit à aller trop loin et, finalement, à répondre à la question initiale de $\mathrm{M}(05 \mathrm{a})$ alors même qu'il l'avait d'abord soigneusement évitée par le recours à une question miroir (06). Ainsi, en guidant les enfants, An a réalisé une structure d'assistance que les enfants n'ont pas refusée, ce qui n'est pas étonnant puisqu'ils l'ont même sollicitée, et qu'ils ont, de fait, acceptée.

Il est toutefois important de souligner le comportement de $\mathrm{C}$ en 18. En pointant la commande de synchronisation avec la souris, C 'interrompt' An par une action en indiquant clairement qu'il sait ce qu'il faut faire à présent. Et c'est précisément la première opération concernant l'activité coopérative qui est concernée. Comme si, dès lors qu'il s'agit de manipuler la plateforme Coopéra, celle qui est au cœur de l'activité scolaire du jour, C manifestait le besoin de reprendre le contrôle. Mieux, $\mathrm{C}$ fait plus qu'une proposition d'action que An viendrait valider 
(20a). En pointant l'icône de synchronisation au moyen du curseur, elle montre en effet qu'elle est 'sur le point' de réaliser l'action en question.

\subsubsection{Centration sur la procédure versus centration sur la coopération}

Malgré tout, il aura fallu que $\mathrm{C}$ ait été dégagée de la procédure liée au traitement de texte et au système d'exploitation pour reprendre la main sur la procédure coopérative. Autrement dit, cette séquence suggère que les élèves ont une compréhension faible de la situation sans l'aide de l'animateur. Cette faiblesse va dans le sens d'une non-prise en considération de l'autre du fait de la centration sur les procédures. Centration sur les procédures qui est encore bien marquée dans l'intervention 20b, lorsque C souligne qu'il "faut faire le contraire ». Le contraire de quoi ? De ce que les enfants ont réalisé en début de séance, c'est-à-dire de la mise à jour de son fichier : une synchronisation d'abord (qui fonctionne dans les deux sens et qui, de toute façon n'est symbolisée que par un seul signe), puis une publication, qui est l'inverse de la mise à jour. En ce sens, $\mathrm{C}$ a raison. En effet, en disant qu'il "faut faire le contraire », $\mathrm{C}$ montre qu'elle maîtrise les fonctions de partage de fichiers.

Toutefois, en disant cela, $\mathrm{C}$ fait référence au déplacement des fichiers, d'espaces à espaces, et non à une intention coopérative. Faire œuvre de coopération, ce ne serait pas faire le contraire, mais faire la même chose, c'est-à-dire partager un fichier modifié avec les autres membres du groupe. Ainsi, en se centrant sur la procédure, C confirme qu'elle n'est pas inscrite dans un processus de coopération, mais plus simplement dans une procédure qui ne nécessite pas (ou peu) de prendre autrui en considération. L'animation que nous proposons devra prendre cela en compte.

\subsubsection{Une animation pour l'acculturation au mode de coopération porté par la plate-forme}

Dans la séance dont nous venons d'étudier un extrait, les élèves n'ont fait aucune référence à autrui. Elles n'ont fait que s'inscrire dans une procédure type, à suivre à chaque utilisation de la plate-forme. Celle-ci est alors utilisée par les élèves comme instrument technique, c'est-àdire essentiellement comme instrument de médiation avec l'objet de leur activité : la réalisation du magazine en ligne. Ce déficit est le signe selon nous d'une non-intériorisation du mode de coopération porté par la plate-forme, résultant d'une rupture entre celui-ci et les modes de coopération 'classiques' à leur environnement culturel habituel. Cette hypothèse d'une rupture culturelle de la dynamique relationnelle visée à travers l'usage de la plate-forme est renforcée par un sondage réalisé au début du projet auprès des enfants au sujet de leurs représentations concernant le travail à distance. Les élèves avaient alors pour seule référence à ce moment là le travail synchrone et plus précisément l'échange de courriers électroniques. Ce déficit s'explique également par le processus même de prise de conscience par les élèves des fonctions visées, au sens où celui-ci ne s'effectue pas d'emblée. En effet, comme le souligne Bruner (Bruner, 2000), en référence aux conceptions vygotskyennes, toute prise de conscience de fonctions n'a lieu qu'après que celles-ci aient été acquises et manipulées.

\subsubsection{Quand et comment réaliser l'animation : deux dimensions fondamentales de son efficacité dans le processus d'acculturation}

Selon le diagnostic ci-dessus, la seule issue au déficit mis en évidence repose sur la mise en œuvre d'une micro-genèse sociale des élèves. Celle-ci se caractérise principalement par l'appropriation par ceux-ci du mode relationnel propre à la plate-forme, dont les concepts centraux sont prévenir et partager. L'objectif visé à travers ce processus est que les élèves prennent conscience de leur rôle au sein de la dynamique coopérative. Cela se traduira par 
leur capacité à mener, à chaque situation rencontrée, une pensée réflexive relative à leur relation avec les autres membres de leur équipe avant d'accomplir ou non une action.

Cette micro-genèse sociale a été initiée lors de la première animation réalisée dans chaque classe. Comme cela a été énoncé dans le diagnostic, la prise de conscience qui conduira les élèves à cette pensée réflexive ne peut s'effectuer d'emblée. Or, à la suite des trois premières séances d'utilisation, les élèves manipulent efficacement les différentes fonctions relatives au mode de coopération propre à la plate-forme. C'est pourquoi, nous avons jugé pertinent de réaliser à cet instant une animation dans chaque classe, dans l'objectif d'étayer chez les élèves la prise de conscience recherchée.

Quant à la forme de l'animation, elle a consisté en une mise en scène concrète de l'activité coopérative propre à la plate-forme. Les notions que les élèves doivent acquérir y sont portées par deux dispositifs matériels : une animation Powerpoint ${ }^{\circledR}$ et un poster qui restera affiché dans chaque classe pour la suite de la réalisation du magazine en ligne. Outre le fait de pallier la rupture culturelle du mode de coopération porté par la plate-forme, une telle animation, parce qu'elle instaure une dynamique entre les différents acteurs, conduit les élèves à être d'avantage motivés à participer au projet et à prendre conscience plus facilement des principes de coopération. En effet, leur engagement dans l'activité collective, ainsi que l'intériorisation des différentes notions abordées dépendent de leur expérience de l'animation, qui ne doit pas être essentiellement cognitive, mais également émotionnelle.

\subsubsection{Les éléments-clé de l'animation}

L'animation s'est déroulée en quatre étapes. Les deux premières ont été réalisées sans référence explicite à la plate-forme en tant qu'outil technique. Ainsi, nous avons commencé par faire un bilan avec les élèves sur leurs connaissances relatives au projet lui-même et à la notion de conflit. Il est alors apparu que les élèves avaient une bonne maîtrise de ces deux points. Au cours de la deuxième étape, nous sommes entrés dans le vif du sujet. Après que les élèves ont indiqué qu'il y a eu des conflits lors des premières séances d'utilisation, nous leur en avons rappelé la cause : l'absence de prise en considération des autres membres de l'équipe. Nous avons enchaîné sur les principes selon lesquels il est essentiel, lorsque l'on est en relation avec d'autres personnes, de réfléchir sur l'impact de nos actions sur autrui et de ne pas juger hâtivement les actions d'autrui envers soi. Ces principes renvoient à la notion de décentration, qui est centrale dans toute activité coopérative.

Nous avons alors abordé les aspects techniques de la plate-forme au cours des deux étapes suivantes. Ainsi, lors de la troisième étape, nous avons précisé aux élèves que les principes de coopération explicités sont également valables lorsqu'on réalise un projet avec d'autres personnes à l'aide de la plate-forme. L'objectif de cette troisième étape était qu'ils prennent conscience de la nécessité de prendre autrui en considération au cours de leurs activités via le collecticiel et in fine du rôle de chaque espace en terme de relation entre soi et autrui. Enfin, la quatrième étape a consisté en une simulation des situations au cours desquelles les élèves devaient adopter ces principes.

Du point de vue de la forme des instruments utilisés, nous avons repris les icônes qui figurent sur le poste de pilotage, l'interface dédiée à la gestion de l'activité coopérative. La cohérence de forme entre les matériaux utilisés lors de l'animation et l'interface de la plate-forme est essentielle pour assurer une continuité entre l'animation et les séances d'utilisation suivantes. Nous faisons référence à Vygotsky lorsqu'il défend que la conscience de soi est contact social avec soi-même (1978) pour élaborer quatre situations qui permettent de présenter à la fois la propre vue de l'élève et celle des autres membres de l'équipe (Figure 3). Ainsi, nous avons commencé par demander aux élèves ce qu'il fallait faire dans la situation présentée (1). Puis nous leur avons demandé quel espace devait être activé dans la visualisation des autres (sur le poste de pilotage) pour que ceux-ci perçoivent leur intention d'action (2). Par la suite, les 
élèves devaient dire quelles étaient les opérations à réaliser pour que cet espace soit effectivement activé sur le poste de pilotage de leurs camarades distants (3). Enfin, ils devaient dire quel serait l'espace activé dans leur propre visualisation (4).

" insérer figure 3 ici "

\subsubsection{Séquence post-animation : intériorisation du mode de coopération de la plate-forme}

Revenons maintenant à l'usage, en classe, de la plate-forme par les deux élèves dont nous avons déjà étudié l'activité conjointe. Au moment où commence l'extrait de cette séquence post-animation, $\mathrm{C}$ et $\mathrm{M}$ ont fini de modifier leur fichier. Comme lors de la séance préanimation, elles n'ont fait aucune référence à autrui durant leur travail. En particulier, elles n'ont prévenu aucun membre de leur équipe de leur activité en cours. Leur démarche ne correspond pas alors à celle préconisée (Tableau 3 ).

\section{" insérer tableau 3 ici "}

Cependant, à travers l'analyse de l'extrait ci-dessous, nous allons montré que les deux élèves se sont tout de même acculturées au mode de coopération propre au collecticiel.

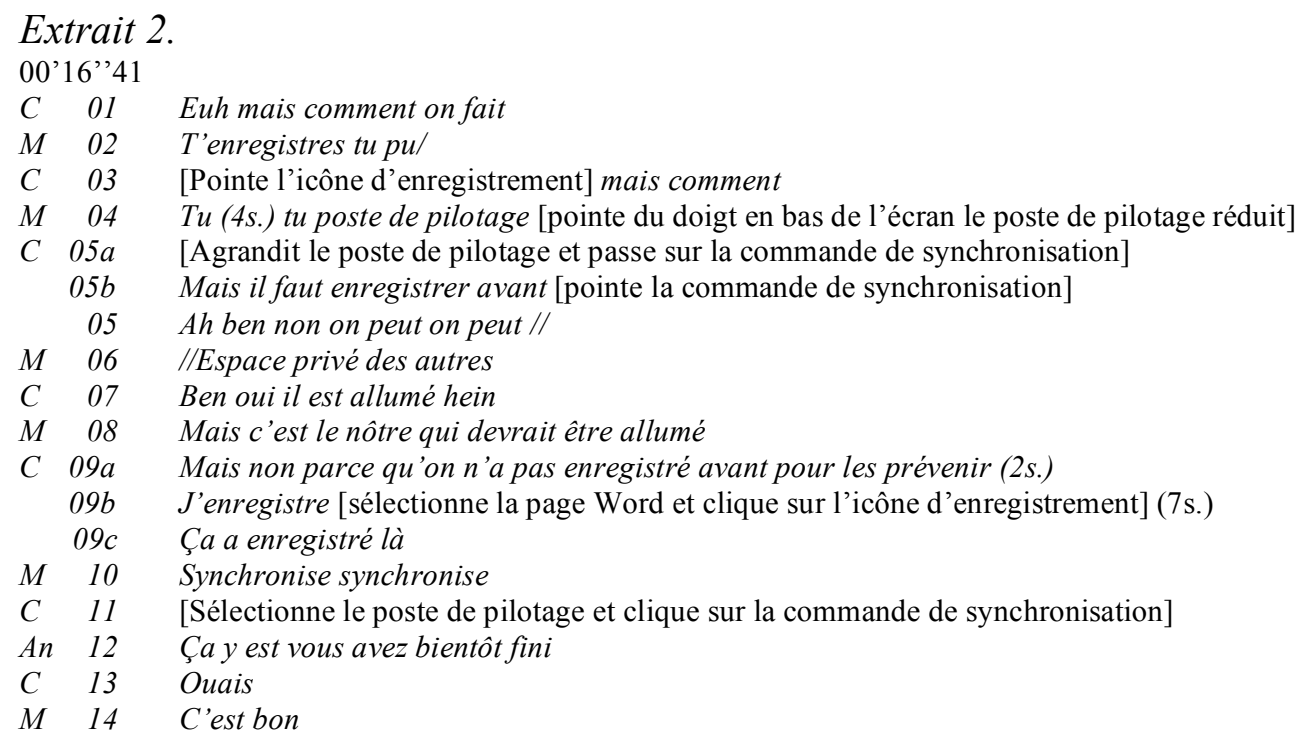

\subsubsection{Manifestation du développement de la conscience de groupe}

La séquence débute par une question de $\mathrm{C}(01)$ sur la manière d'atteindre un objectif : " euh mais comment on fait ". Cette première intervention marque une avancée dans l'usage de la plate-forme par rapport à la séance pré-animation, au sens où $\mathrm{C}$ envisage de procéder à une manipulation de leur fichier. Cependant, elle ne donne aucune précision sur son intention. Deux hypothèses peuvent être alors posées.

- C souhaite simplement déplacer leur fichier dans un espace autre que leur espace local. Elle ferait donc référence à la procédure d'utilisation à suivre dans cette situation ;

- C souhaite réaliser une action à l'intention des autres membres de son équipe. Auquel cas, on pourrait voir dans cet énoncé un début de développement de la conscience de groupe.

Or, comme nous l'avons montré dans l'analyse du premier extrait, $\mathrm{C}$ maîtrise les fonctions de partage de fichiers. En d'autres termes, elle connaît la procédure pour le déplacement des 
fichiers d'espace à espace. Ainsi, à défaut de pouvoir l'affirmer, nous posons que son énoncé est l'expression d'une référence aux autres binômes de l'équipe.

La suite des échanges entre $\mathrm{M}$ et $\mathrm{C}$ jusqu'en $\mathrm{C} 05 \mathrm{~b}$ consiste en une description conjointe des opérations à réaliser pour atteindre l'objectif recherché : il faut enregistrer le fichier, puis ouvrir le poste de pilotage pour opérer une synchronisation. Cette démarche revient à placer leur fichier dans leur espace privé. Si à travers ces échanges les deux élèves expriment leur objectif, nous ne pouvons toujours pas conclure si celui-ci est d'ordre procédural ou d'ordre épistémique. Cependant, au regard de la séance pré-animation où $\mathrm{C}$ n'avait comme seule référence la procédure consistant à déplacer un fichier de l'espace commun à leur espace local et inversement, le fait qu'elles envisagent de transférer leur fichier dans leur espace privé et non dans l'espace commun appuie l'hypothèse selon laquelle leur action est orientée vers les autres élèves. Action qui serait alors de les prévenir, ce qui à cet instant n'est pas pertinent puisqu'elles ont terminé leur travail.

À la vue du poste de pilotage, où seul l'espace privé d'autrui est activé, M 08 objecte que ce n'est pas celui-ci qui devrait être activé, mais le leur ("mais c'est le nôtre qui devrait être allumé »), alors qu'elles n'ont fait aucune modification à leur écran. Si l'énoncé de M est l'expression de son incompréhension face à la situation présente, il est cependant intéressant pour le binôme puisqu'elle amène $\mathrm{C}(09 \mathrm{a})$ à expliciter l'objectif recherché : «... les prévenir ». Notre hypothèse concernant leur intention est alors confirmée : $\mathrm{M}$ et $\mathrm{C}$ ont développé une pensée réflexive à l'égard d'autrui.

L'intervention de M 08, contextualisée par la présence du poste de pilotage sur leur écran d'ordinateur, a également conduit $\mathrm{C}$ à effacer toute hésitation concernant la démarche à suivre. Cela se traduit par la mise en œuvre effective de celle-ci (C09b à M14). À cet instant, nous pouvons affirmer que $\mathrm{M}$ et $\mathrm{C}$ ont stabilisé conjointement le sens quant à la démarche à suivre pour prévenir autrui.

\subsubsection{Autonomie des élèves vis-à-vis de l'animateur}

Une autre marque de l'appropriation par les deux élèves des principes du mode de coopération permis par la plate-forme est leur autonomie vis-à-vis d'An. En effet, elles n'ont en aucun moment fait appel à An au cours de l'usage du collecticiel pour la réalisation de leur projet, contrairement à la séance pré-animation. Pourtant, il aurait été justifié qu'elles le sollicitent alors qu'elles hésitaient dans la définition de la démarche à suivre pour prévenir les autres membres de leur équipe. Ceci confirme selon nous leur aisance quant au modèle de partage de fichiers.

Leur autonomie vis-à-vis d'An est le signe aussi que celui-ci a pleinement joué son rôle dans ce processus d'appropriation par les élèves du mode de coopération propre à l'outil. En effet, il a mis en place le dispositif pédagogique nécessaire pour que ce processus se réalise effectivement et a géré les interactions en fonction des différentes phases d'usage dans lesquelles elles se situent.

\subsubsection{Intériorisation du mode de coopération}

L'attitude des deux élèves envers les autres membres de leur équipe est la trace de l'animation réalisée précédemment dans chaque classe. Cependant, deux questions subsistent. Premièrement, pourquoi $\mathrm{M}$ et $\mathrm{C}$ n'ont-elles manifesté cette intention de prévenir autrui qu'à la fin de leur travail ? Deuxièmement, pourquoi ont-elles un peu hésité dans la détermination de la démarche à suivre pour prévenir autrui, alors qu'elles connaissent les principes de fonctionnement du modèle de partage de fichiers?

$\mathrm{M}$ et $\mathrm{C}$ ont fourni une réponse à la première question lors d'un entretien en auto-confrontation réalisé juste après cette séance. L'oubli de prévenir les autres lors de leur travail en cours 
serait tout simplement dû au fait que " prévenir [elles] ne l'avaient jamais fait avant ». Quant à la seconde question, nous expliquons leur hésitation par l'accomplissement d'un processus de restructuration de leurs connaissances quant au modèle de partage de fichiers. Celles-ci ne sont plus seulement mobilisées pour l'orientation et l'accomplissement de leur activité, mais également pour la compréhension des situations. Autrement dit, l'usage que les élèves font de la plate-forme est dorénavant caractérisé par trois dimensions : heuristique, procédurale et épistémique. Cette restructuration marque l'intériorisation par les deux élèves du mode de coopération permis par la plate-forme. L'aboutissement de ce processus est confirmé par l'explicitation faite par $\mathrm{C}$ au cours de l'entretien en auto-confrontation à propos de la démarche à suivre pour prévenir les autres : "Ben on enregistre et après on on on le on le synchronise pour le mettre dans notre espace privé et le chez les chez eux ça enfin sur leur ordinateur ça sera marqué espace privé des autres allumé ». A cet instant, la plate-forme n'est plus seulement utilisée par les élèves comme instrument technique, mais également comme instrument psychologique, c'est-à-dire, comme instrument de médiation avec l'objet de leur activité, avec les autres et avec eux-mêmes.

\section{Discussion}

L'animation présentée dans cet article est centrale dans le processus de prise de conscience par le binôme des principes de coopération propres à la plate-forme. Précisons que nous retrouvons les mêmes résultats chez les autres groupes d'élèves que nous avons suivis. Cependant, la résonance d'une telle animation sur l'usage du collecticiel va cependant bien au-delà de son rôle dans ce processus. En effet, d'une part, sa mise en place comme momentclé dans le processus d'appropriation de la plate-forme nous a conduit à reconsidérer l'intérêt pédagogique de l'environnement informatique. D'autre part, elle s'est révélée efficace dans le cycle de conception de la plate-forme. Ce sont ces deux points que nous allons développer en discussion.

\subsection{L'appropriation de la plate-forme : un intérêt pédagogique primordial}

Les difficultés des élèves dans l'appropriation de la plate-forme résident principalement dans l'acculturation aux principes de coopération spécifiques à l'outil informatique. Ces difficultés peuvent être perçues comme une contrainte si l'on envisage la conscience de groupe comme une condition sine qua non à l'usage de la plate-forme. En termes ergonomiques, ces difficultés relèveraient dès lors d'un problème d'acceptabilité, c'est-à-dire d'une incompatibilité entre la culture des élèves (et des enseignants) et l'usage permis par le collecticiel. Or, comme nous l'avons montré dans cet article, l'appropriation par les élèves de la plate-forme, qui se traduit par leur capacité à mener une pensée réflexive quant à leur rôle au sein de la dynamique coopérative, résulte d'un processus complexe, caractérisé par des phases d'usage de natures différentes, ainsi que par des moments d'amorce et d'étayage de la prise de conscience visée (Figure 4).

\section{"insérer figure 4 ici "}

Aussi, outre la réalisation du projet, l'objectif recherché tout au long de l'utilisation de la plate-forme est que les élèves développent cette capacité à se décentrer d'eux-mêmes et à réfléchir systématiquement sur l'impact de leurs conduites vis-à-vis d'autrui à chaque situation rencontrée. La conscience de groupe n'est donc pas la condition sine qua non à l'usage du collecticiel, mais bien l'objet visé via l'usage de celui-ci. En d'autres termes, les difficultés des élèves ne relèvent pas du critère d'acceptabilité, mais de celui d'utilité, c'est-à- 
dire de l'objectif d'apprentissage visé à travers l'usage du collecticiel. Un des intérêts pédagogiques majeures de l'outil est donc de conduire les élèves à s'approprier les fondements de l'altérité, essentiels dans toute situation de travail coopératif. L'outil n'est alors pas seulement une ressource pour mener à bien leur projet, mais également une source qui inscrit les élèves dans un mode de coopération, dont la nouveauté permet de mettre à jour plus facilement la complexité des relations inter-personnelles.

\subsection{Le rôle de l'animation dans le cycle de conception}

Perçue comme fondamentale dès lors que l'analyse des interactions entre enfants a permis de montrer une difficulté d'appropriation de Coopéra bien plus conceptuelle qu'opératoire, l'animation a d'abord été conçue comme un moyen pour permettre aux élèves de prendre conscience des principes du mode coopération propres à la plate-forme. Cet objectif était pleinement explicite lors de sa mise en place au cours de la réalisation du projet. Mais cette animation s'est également révélée efficace pour une autre raison, tout aussi fondamentale. En effet, elle s'est révélée être une méthode d'évaluation ergonomique permettant de faire des préconisations sur la conception. Nous commencerons par montrer l'importance de cet aspect de l'animation quant à notre position dans le cycle de conception et plus précisément dans l'élaboration de la troisième version de la plate-forme. Puis, nous présenterons deux améliorations à apporter au poste de pilotage.

\subsection{1 'Rendre compte de' ou 'faire des propositions' : l'animation comme réponse efficace au temps nécessaire pour nos analyses}

En raison du temps nécessaire pour nos analyses, nous sommes confrontés au problème selon lequel nous ne pouvons pas pleinement exploiter les retours que nous faisons sur les propriétés ergonomiques d'une version V1 de la plate-forme pour le développement de la version V2 du collecticiel mais pour sa version V3. Aussi, comme cela est représenté dans la figure 6 ci-dessous, les analyses des corpus recueillis à la suite du premier projet [C1] ont servi à l'élaboration de la troisième version de la plate-forme [PF3] et non à celle de la deuxième version [PF2]. Celle-ci a été essentiellement réalisée par l'équipe de développement [D1], sur la base tout de même d'échanges que nous avons eus avec elle, mais pas sur la base d'analyses formelles. L'efficacité de l'animation réside précisément en ce qu'elle nous permet de dépasser cette difficulté liée à notre méthodologie d'analyse. En effet, grâce à l'animation, nous avons été en mesure d'évaluer rapidement une version de la plateforme et de faire des préconisations ergonomiques pour le développement de la version suivante. C'est ainsi que les animations réalisées au cours du second projet [A2], nous ont permis d'évaluer la seconde version de plate-forme [PF2], de faire des propositions d'améliorations ergonomiques pour la troisième version du collecticiel [PF3] et de travailler en étroite collaboration avec l'équipe de développement [D2] en amont de son élaboration.

\section{" insérer figure 5 ici »}

\subsubsection{Deux améliorations ergonomiques à apporter au poste de pilotage}

A la suite de cette animation, nous avons relevé deux améliorations majeures à apporter au poste de pilotage. Elles concernent la visualisation, ainsi que le code couleur. 


\subsubsection{Une visualisation plus représentative de l'intentionnalité des élèves des uns envers les autres}

La visualisation figurant sur la version de plate-forme utilisée par les élèves représente le modèle de partage de fichiers. Cette visualisation leur permet certes de ne pas fournir d'efforts cognitifs trop importants lorsqu'ils doivent décider des opérations à réaliser pour atteindre un objectif. La plate-forme est alors un support favorable à l'activité de représentation, au sens de rendre présent des significations, relative à la co-réalisation des fichiers propres à chaque équipe interclasse. Cependant, ce qui prime dans l'usage de la plate-forme, c'est la relation à l'autre. Dans cette perspective, les élèves n'ont pas à s'approprier le modèle de partage de fichiers sous-jacent au mode de coopération, mais seulement le mode de coopération luimême. Aussi, la visualisation actuelle doit être remplacée par une visualisation plus explicite et culturellement et du point de vue de la dynamique relationnelle visée. Plus précisément, parmi les icônes représentant les différents espaces, celle qui nécessite absolument d'être modifiée est l'espace privé, qui rappelons-le sert à prévenir qu'un travail est en cours. Elle pourrait être remplacée par exemple par un feu clignotant, dont le faisceau serait dirigé vers la personne visée. En revanche, l'espace local (qui pourrait être renommé 'privé' et prendre la forme d'un ordinateur) et l'espace commun doivent être préservés pour signifier la différence entre soi et le groupe. Ces modifications ne peuvent prendre sens que dans la mesure où le système de commandes de partage de fichiers y est en adéquation. En effet, le système actuel induit trop fortement la notion d'espace. Par exemple, lors d'une mise à jour, le fichier provenant de l'espace commun n'arrive pas directement dans l'ordinateur, mais passe avant par 'quelque part' (l'espace privé). Ainsi, changer l'icône représentant l'espace privé et préserver les commandes actuelles de partage de fichiers serait insensé, au sens où un feu clignotant ne peut ni contenir un fichier ni être synchronisé avec un espace (local). La plateforme serait alors un support favorisant l'activité de représentation relative et à la coréalisation des fichiers propres à chaque équipe interclasse et à la régulation de l'activité coopérative.

\subsubsection{Le code couleur est-il toujours nécessaire?}

A l'origine, le code couleur était la seule visualisation de l'activité coopérative. Depuis, la visualisation a considérablement évolué, mais il ne fut cependant pas question d'abandonner ce code couleur au moment de son développement. En effet, lors des réflexions entre équipes d'usage quant à la mise au point de la visualisation, il était prévu de situer les ronds de couleur dans les espaces correspondants. Ainsi, seuls les ronds devaient indiquer d'une part l'apparition d'un nouvel événement et d'autre part l'espace concerné. Cependant, pour des raisons techniques, nous avons dû séparer les ronds de la visualisation et doter cette dernière de son propre système d'indication. Dès lors, le code couleur est inutilement redondant car non seulement il n'est plus nécessaire pour comprendre la situation présente, mais en outre il est source de difficultés dans son interprétation par les élèves.

\section{Conclusion}

Concevoir une machinerie informationnelle destinée à permettre à des sujets humains de collaborer n'est pas qu'un travail de développeurs (un travail qui serait effectué en amont de l'usage par les acteurs et qui repousserait l'étude de cet usage dans l'après-coup de sa réalisation technique). Cette constatation devient maintenant banale même si on ne se donne pas toujours les moyens d'assurer une sérieuse prise en compte de cette dialectique 
conception-usage. Nous avons voulu ici proposer un exemple de travail interdisciplinaire abordant cette question de front.

Sur la base d'un premier prototype de la plate-forme, l'analyse de l'usage en situation naturelle, menée d'un point de vue ethnographique, a conduit à des modifications significatives de l'architecture du collecticiel. La mise en œuvre, d'une part, de trois versions de l'outil informatique, et d'autre part, de situations pédagogiques et d'animations impliquant les enseignants et les élèves, montrent bien l'interrelation profonde des pratiques de recherche-développement des informaticiens et des psychologues dans notre projet. Plus, les contenus des versions successives découlent directement des résultats des analyses de l'usage de l'objet numérique par les enfants. Ces résultats touchent à trois facettes de cet usage. En travaillant à la façon dont les enfants appréhendaient cette nouvelle technologie d'information et de communication, trois niveaux nous sont en effet apparus importants : celui du rapport direct avec les fonctionnalités techniques de l'outil, celui de l'inscription dans des scénarios pédagogiques et celui de l'arrière-plan culturel de son utilisation. En utilisant le mot " appropriation » dans le titre de ce texte, nous avons employé un terme relativement neutre et à large spectre. Ce faisant, nous proposons de décliner cette 'appropriation' en trois notions respectivement associées à ces trois niveaux : utilisabilité, utilité et acceptabilité. Au-delà de cette suggestion terminologique, nous voulons soutenir qu'il est bien délicat et peut-être illusoire de prétendre savoir les distinguer finement. Les analyses que nous avons conduites demandent bien sûr à être multipliées afin d'espérer en tirer des régularités. Il nous semble cependant qu'elles permettent d'illustrer le fait que ces trois critères relatifs à l'appropriation d'un dispositif numérique par des collectifs d'humains doivent être évalués de façons interdépendantes et aussi, et peut-être surtout, que cette évaluation doit s'effectuer via une mise en œuvre effective par les acteurs, en situation naturelle. En effet, seule cette mise en situation pourra montrer que ces instruments numériques sont des ressources au travail collaboratif mais qu'ils en sont aussi des sources.

\section{Remerciements}

Nous remercions la société Jériko, nos partenaires académiques (projet ECOO du Loria et équipe Gr@mmsci de l'Université M. de Montaigne, Bordeaux 3), ainsi que Pascal Pierre, conseiller TICE de l'inspection académique de Nancy-Metz, et les quatre écoles qui ont participé à cette étude : SaintPierre et Jules Ferry à Nancy, Louis Pergaud à Laxou et La Sapinière à Toul.

\section{Bibliographie}

Baker, K., Greenberg, S., \& Gutwin, C. (2002). Empirical Development of a Heuristic Evaluation Methodology for Shared Workspace Groupware. Proceedings of CSCW'2002 (pp. 96-105). New Orleans, Louisiana, November 16-20.

Brassac, Ch. (2004). Action située et distribuée et analyse du discours: quelques interrogations. Cahiers de Linguistique Française 26, 251-268.

Brassac, Ch., \& Grégori, N. (2003). Une étude clinique de la conception collaborative : la conception d'un artefact. Le Travail Humain, 66, 101-127.

Brassac, Ch., \& Grégori, N. (2001a). Situated and Distributed Design of a Computer Teaching Device. Journal of Design Sciences and Technology, 8/2, 11-31.

Brassac, Ch., \& Grégori, N. (2001b). La communication en environnement virtuel. Champ psychosomatique, 22, 83-97.

Bruner, J. (2000). Culture et modes de pensée. L'esprit humain dans ses oeuvres. Paris : Retz. 
Cardon, D. (1997). Les sciences sociales et les machines à coopérer. Une approche bibliographique du Computer Supported Cooperative Work (CSCW). Réseaux, 85, 3-39.

Callon, M., Lascoumes, P., \& Barthe, Y. (2001). Agir dans un monde incertain. Essai sur la démocratie technique. Paris : Éditions du Seuil.

Clot, Y. (2003). Vygotski, la conscience comme liaison. In Vygotski L.S. Conscience, inconscient, émotions. Paris : La Dispute.

Clot, Y. (1999). La fonction psychologique du travail. Paris : Presses Universitaures de France.

Clot, Y., Faïta, D., Fernandez, G., \& Scheller, L. (2001). Entretiens en autoconfrontation croisée : une méthode en clinique de l'activité. Education permanente, 146, 17-25.

Conein, B., \& Jacopin, É. (1994). Action située et cognition : le savoir en place. Sociologie du travail, $X X X V I, 475-499$.

Dourish P. (2003). The Appropriation of Interactive Technologies: Some Lessons from Placeless Documents. Computer Supported Cooperative Work, 12, 465-490.

Dourish, P. (1998). Using meta-level techniques in a flexible toolkit for CSCW applications. ACM Transactions on Computer Human Interactions, vol. 5, no. 2.

Engeström, Y., Miettinen, R., \& Punamäki, R.L. (1999). Perspectives on Activity Theory. Cambridge : Cambridge University Press.

Feiler, P.H. \& Downey, G.F. (1990). Transaction-Oriented Configuration Managment: A Case Study. CMU/SEI-90-TR-23 ESD-90/TR-224.

Godart, C., Charoy, F., Patten, M., Grégori, N., Hautecouverture, J.-C., Paquelin, D., \& Faugeras, I. (2003). Coopéra: Apprendre à coopérer et apprendre en coopérant. Proceedings of ICOOL'2003 (International Conference on Open and Online Learning). Mauritius, December 7-13.

Grégori, N. (2002). La conception assistée par l'usage des nouveaux dispositifs : un point de vue interactionniste. Éducation permanente, 152, 121-132.

Hautecouverture, J.-C., Grégori, N., Charoy, F., Godart, C., Patten, M., \& Faugeras, I. (2003). Coopéra: Analyse de l'usage d'une plate-forme de coopération à destination d'enfants du primaire. Human Centered Process Conference - HCP'2003. Luxembourg, 5-7 mai.

Hautecouverture, J.-C., Grégori, N., Paquelin, D., Charoy, F., Godart, C., Patten, M., \& Faugeras, I. (2004). Analyse d'usage d'une plate-forme de coopération : Usage et développement logiciel. In Cognito - Cahiers Romans de Sciences Cognitives, 3, 4577.

Hutchins, E. (1995). Cognition in the Wild. Cambridge: MIT Press.

Jeantet, A. (1998). Les objets intermédiaires dans les processus de conception des produits, Sociologie du travail, 3/98, 291-316.

Kaptelinin, V., \& Nardi, B. (2003). Post-Cognitivist HCI: Second Wave Theories (Panel report) - CHI 2003. Ft. Lauderdale, Florida, April 5-10.

Latour, B. (1999). Politiques de la nature. Comment faire entrer les sciences en démocratie. Paris : Éditions de la Découverte. (Réédition 2004, La Découverte/Poche).

Levinson, S. (1983). Pragmatics. Cambridge : Cambridge University Press.

Mallein, Ph., \& Panisset, J. (1997). Recherche interactive CAUDOC. Conception par l'usage du serveur documentaire Rédoc. Grenoble, Document interne.

Nardi, B., Gilbert, N., Mantei, M, \& McCarthy, J. (1993). Mixing oil and water? Ethnography versus Experimental Psychology in the study of computer-mediated communication. Proceedings of INTERCHI'93 (pp. 3-6), April 24-29.

Rabardel, P. (1999). Le langage comme instrument ? Éléments pour une théorie instrumentale élargie. In Y. Clot (Ed.), Avec Vygotski (pp. 241-265). Paris : La Dispute.

Roulet, E., Auchlin, A., Moeschler, J., Rubattel, C., \& Schelling, M. (1985). L'articulation du discours en français contemporain. Berne : P. Lang. 
Searle, J.R., \& Vanderveken, D. (1985). Foundations of illocutionary logic. Cambridge: Cambridge University Press.

Suchman, L. (1987). Plans and situated actions. Cambridge : Cambridge University Press.

Vanderveken, D. (1988). Les actes de discours. Bruxelles : Mardaga.

Vygosky, L.S. (1978). Mind in Society: The Development of Higher Psychological Process. Cambridge: Harvard University Press. 
Figures et tableaux

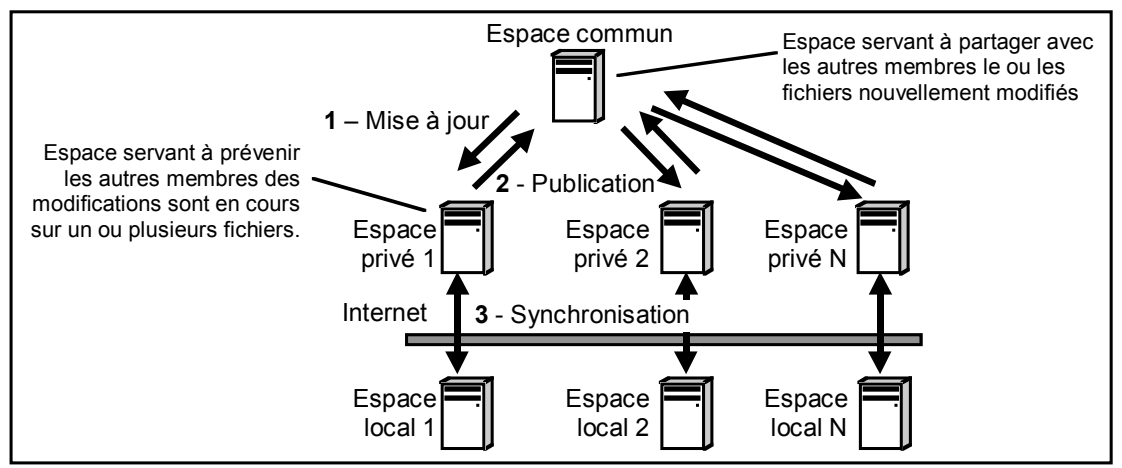

Figure 1. Modèle de partage de fichiers.

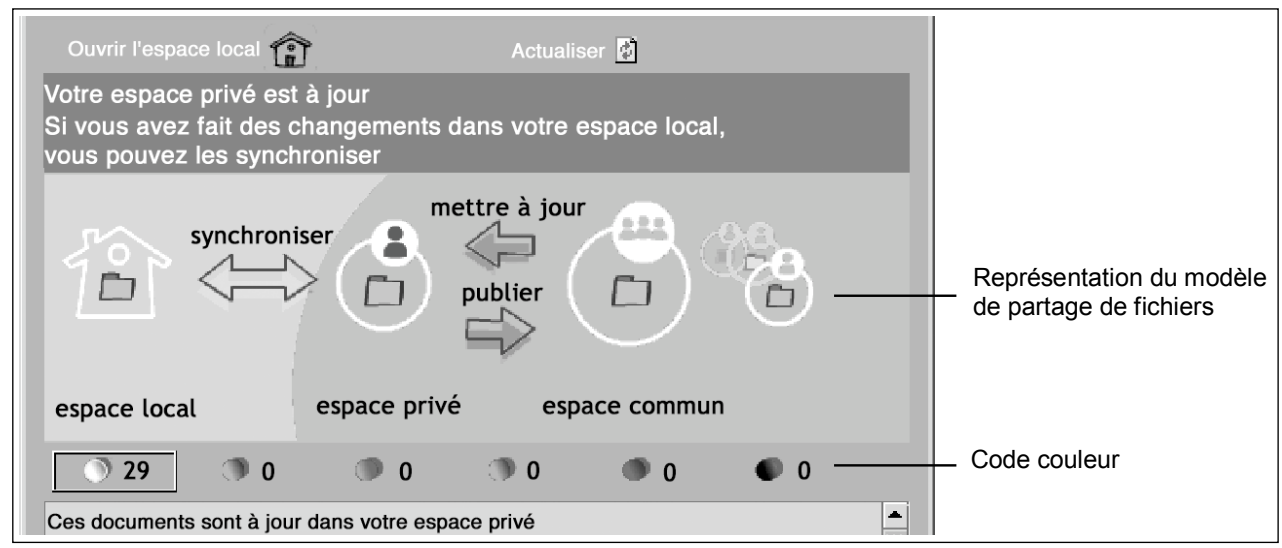

Figure 2. Visualisation de l'activité coopérative. 


\begin{tabular}{|c|c|c|}
\hline & Écrans à l'ordinateur & Caractéristiques d'usage \\
\hline \multirow{4}{*}{$\begin{array}{l}\text { Début du } \\
\text { travail sur } \\
\text { le fichier }\end{array}$} & $\mathrm{TdT}$ & $\begin{array}{l}\text { 1. Interprétation de la situation } \\
\text { - Début du travail sur le fichier. Situation potentiellement } \\
\text { conflictuelle : il est conseillé de prévenir les autres membres du projet }\end{array}$ \\
\hline & \begin{tabular}{l|l}
$\operatorname{PdP}$ \\
$\mathrm{PdP}$
\end{tabular} & $\begin{array}{l}\text { 2. Détermination des actions } \\
\text { - Détermination de l'action } \\
\text { - Ouvrir le poste de pilotage et déposer le fichier dans mon espace } \\
\text { privé } \\
\text { - Détermination de la démarche d'action } \\
\text { - Fermer les fenêtres actuelles (pages Word et espace local), puis } \\
\text { synchroniser }\end{array}$ \\
\hline & \multirow{2}{*}{ espace local } & $\begin{array}{l}\text { 3. Réalisation des actions } \\
\text { 31. Ouverture du poste de pilotage } \\
\text { - Localisation des commandes } \\
\text { - Activation des commandes }\end{array}$ \\
\hline & & $\begin{array}{l}\text { 32. Transfert du fichier dans mon espace privé } \\
\text { - Localisation des commandes } \\
\text { - Activation des commandes }\end{array}$ \\
\hline \multirow{4}{*}{$\begin{array}{l}\text { Fin du } \\
\text { travail sur } \\
\text { le fichier }\end{array}$} & \multirow{2}{*}{ TdT } & $\begin{array}{l}\text { 1'. Interprétation de la situation } \\
\text { - Fin du travail sur le fichier. Situation potentiellement conflictuelle : } \\
\text { il est conseillé de partager les autres membres du projet }\end{array}$ \\
\hline & & $\begin{array}{l}\text { 2'. Détermination des actions } \\
\text { - Détermination de l'action } \\
\text { - Ouvrir le poste de pilotage et déposer le fichier dans sa version } \\
\quad \text { finale dans l'espace commun } \\
\text { - Détermination de la démarche d'action } \\
\text { - Fermer les fenêtres actuelles (pages Word et espace local), puis } \\
\text { synchroniser et publier }\end{array}$ \\
\hline & \multirow{2}{*}{ 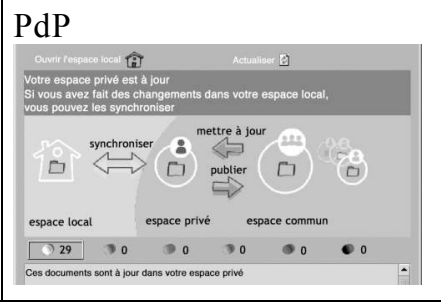 } & $\begin{array}{l}\text { 3'. Réalisation des actions } \\
\text { 31'. Ouverture du poste de pilotage } \\
\text { - Localisation des commandes } \\
\text { - Activation des commandes }\end{array}$ \\
\hline & & $\begin{array}{l}\text { 32'. Transfert du fichier dans mon espace privé, puis dans l'espace } \\
\text { commun } \\
\text { - Localisation des commandes } \\
\text { - Activation des commandes }\end{array}$ \\
\hline
\end{tabular}

Tableau 1. Écrans successifs et caractéristiques d'usage propre à la démarche préconisée en situation de travail sur un fichier.

\begin{tabular}{|c|c|c|}
\cline { 2 - 3 } \multicolumn{1}{c|}{} & Démarche préconisée & Démarche réelle - Séance pré-animation \\
\hline \multirow{4}{*}{$\begin{array}{c}\text { Début du } \\
\text { travail sur le } \\
\text { fichier }\end{array}$} & $\begin{array}{c}\text { 1. Interprétation de la situation } \\
\text { - Début d'un travail sur le fichier. Situation } \\
\text { potentiellement conflictuelle }: \text { il faut prévenir } \\
\text { autrui }\end{array}$ & $\begin{array}{c}\text { 1. Interprétation de la situation } \\
-\varnothing\end{array}$ \\
\cline { 2 - 3 } & $\begin{array}{c}\text { 2. Détermination des actions } \\
\text { - Détermination de l'action } \\
- \text { Déposer le fichier dans l'espace privé }\end{array}$ & $\begin{array}{c}\text { 2. Détermination des actions } \\
\text { - Détermination de l'action } \\
-\varnothing \\
\text { - Détermination de la démarche d'action } \\
\text { - Synchroniser }\end{array}$ \\
\hline
\end{tabular}

Tableau 2. Comparaison entre la démarche d'usage préconisée et celle effective de $M$ et $C$ lors de la séance pré-animation. 


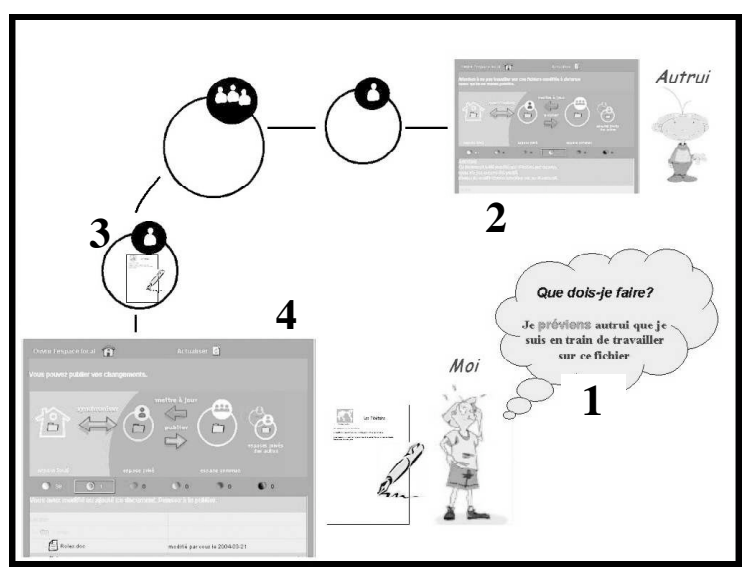

Figure 3. Exemple d'une situation présentée au cours de l'animation.

\begin{tabular}{|c|c|c|}
\hline & Démarche préconisée & Démarche réelle - Séance post-animation \\
\hline \multirow{2}{*}{$\begin{array}{l}\text { Début du } \\
\text { travail sur le } \\
\text { fichier }\end{array}$} & $\begin{array}{l}\text { 1. Interprétation de la situation } \\
\text { - Début d'un travail sur le fichier. Situation } \\
\text { potentiellement conflictuelle : il faut prévenir } \\
\text { autrui }\end{array}$ & $\begin{array}{l}\text { 1. Interprétation de la situation } \\
-\varnothing\end{array}$ \\
\hline & $\begin{array}{l}\text { 2. Détermination des actions } \\
\text { - Détermination de l'action } \\
\text { - Déposer le fichier dans l'espace privé } \\
\text { - Détermination de la démarche d'action } \\
\text { - Synchroniser } \\
\end{array}$ & $\begin{array}{l}\text { 2. Détermination des actions } \\
\text { - Détermination de l'action } \\
-\varnothing \\
\text { - Détermination de la démarche d'action } \\
-\varnothing\end{array}$ \\
\hline
\end{tabular}

Tableau 3. Comparaison entre la démarche préconisée et celle de $\mathrm{M}$ et $\mathrm{C}$ lors de la séance post-animation.

\begin{tabular}{|c|c|c|c|}
\hline $\begin{array}{l}\quad \text { Animation } \\
\text { Présentation des } \\
\text { principes de } \\
\text { coopération et des } \\
\text { fonctions de la plate- } \\
\text { forme : amorce de la } \\
\text { prise de conscience } \\
\text { de ces principes. }\end{array}$ & $\begin{array}{l}\text { Usages de l'outil } \\
\text { Acquisition et } \\
\text { manipulation des } \\
\text { fonctions de la plate- } \\
\text { forme. }\end{array}$ & $\begin{array}{l}\quad \text { Animation } \\
\text { Etayage de la prise de } \\
\text { conscience des } \\
\text { principes de } \\
\text { coopération. }\end{array}$ & $\begin{array}{l}\text { Usages de l'outil } \\
\text { Développement de la } \\
\text { dimension épistémique } \\
\text { des connaissances des } \\
\text { élèves } \alpha \text { émergence de } \\
\text { la conscience de } \\
\text { groupe. }\end{array}$ \\
\hline
\end{tabular}

Figure 4. Les différentes phases du processus d'appropriation de la plate-forme. 


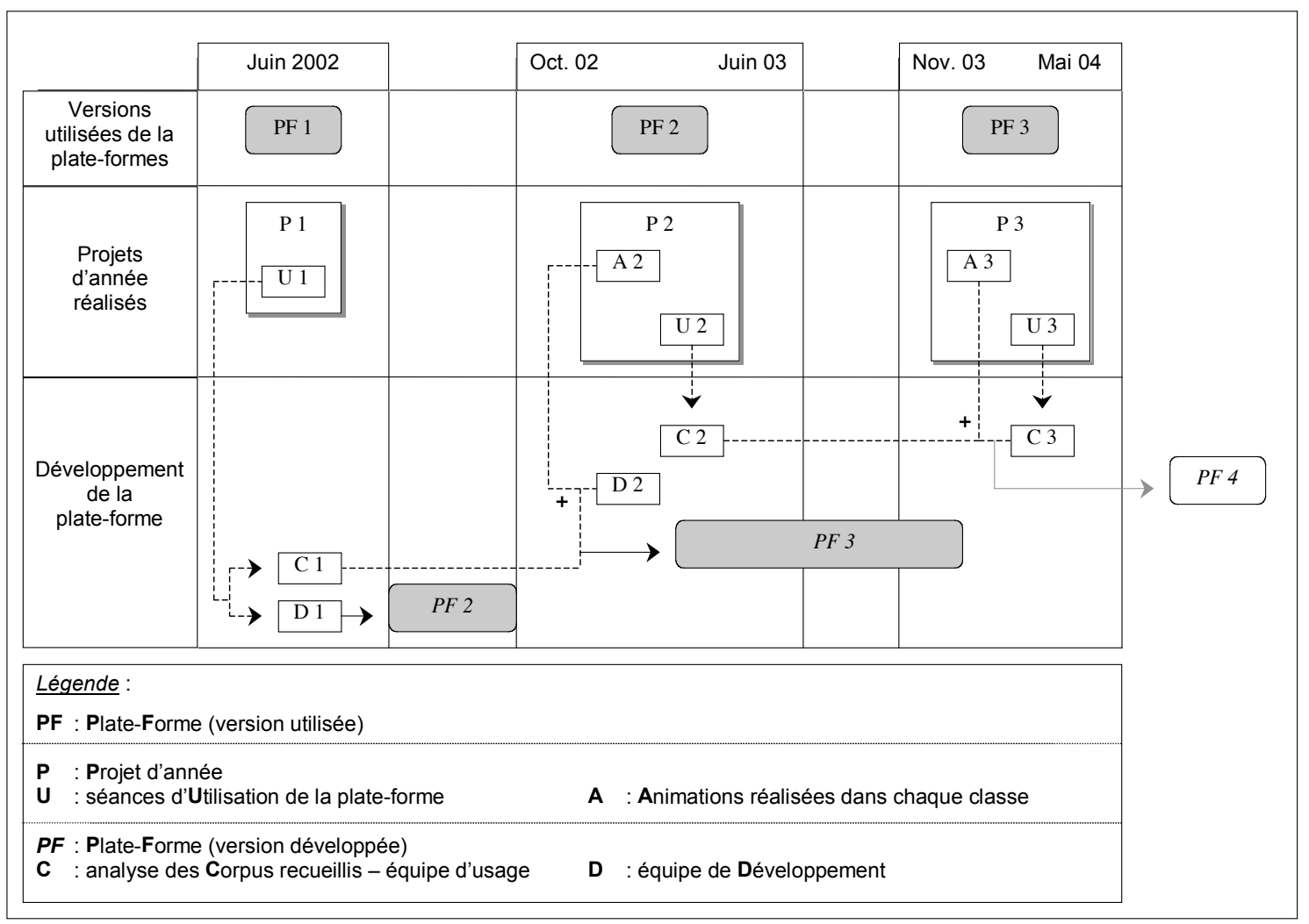

Figure 5. Cycle de conception de la plate-forme Coopéra. 